\title{
Nasal markers of CNS-targeted drug activity
}

Biopsy samples of nasal olfactory epithelium could provide an easyaccess readout of CNS-targeted drug activity, according to a recent study. "A big problem in CNS drug discovery is showing that a drug really works in patients, as mouse studies are in reality not good predictors," says Jeffrey Rothstein, who led the study.

\section{4 a big problem in CNS drug discovery is showing that a drug really works in patients... 77}

Earlier work had shown that rodent nasal olfactory epithelium expresses markers of various CNS cell types, including neurotransmitters and the astrocytic marker glial fibrillary acidic protein (GFAP). Rothstein's group wondered whether this tissue could provide a peripheral pharmacodynamic marker of drug activity in the CNS.

The researchers employed the drug thiamphenicol, which is an upregulator of excitatory amino acid transporter 2 (EAAT2), an astrocytic glutamate transporter. Reduced astrocytic expression of this protein has been observed in a number of neurological disorders, including amyotrophic lateral sclerosis, in which its loss is thought to contribute to glutamate excitotoxicity, leading to neuronal cell death.

Rothstein and colleagues began by performing western blot analysis of mouse and human nasal olfactory epithelium, which confirmed the presence of EAAT2 in this tissue. Treatment with thiamphenicol over 14 days in mice increased EAAT2 levels in the CNS and olfactory epithelium to a similar extent. No such increase was observed in saline-treated animals.

The team then conducted a 14-day phase I placebo-controlled trial of oral thiamphenicol in 30 healthy volunteers, aiming to provide proof of concept for this pharmacodynamic approach. Nasal biopsies were taken 1 day before treatment and on day 14. Cerebrospinal fluid (CSF) samples were collected on day 13 to provide an estimate of the drug concentration in the brain.

The drug dose correlated positively with plasma and CSF drug levels, and showed a trend towards increasing EAAT2 and GFAP expression in the olfactory epithelium. Nasal biopsies could, therefore, provide a rapid and accessible marker of drug activity in the CNS.

Growing interest has been shown in astrocytes as therapeutic targets, owing to their roles in numerous neurodegenerative and psychiatric disorders. "This is the first study of astrocytic biomarkers," says Rothstein. "The findings could be used by other researchers aiming to modify human astroglia."

Katie Kingwell

Original article Sattler, R. et al. Human nasal olfactory epithelium as a dynamic marker for CNS therapy development. Exp. Neurol. doi:10.1016/j.expneurol.2011.09.002 